\title{
The spectroscopy of solar sterile neutrinos
}

\author{
Ilídio Lopes ${ }^{1,2, a}$ \\ ${ }^{1}$ Departamento de Física, Instituto Superior Técnico-IST, Centro de Astrofísica e Gravitação-CENTRA, Universidade de Lisboa-UL, Av. Rovisco \\ Pais 1, 1049-001 Lisboa, Portugal \\ 2 Institut d'Astrophysique de Paris, UMR 7095, CNRS \& UPMC, Sorbonne Universités, 98 bis bd Arago, 75014 Paris, France
}

Received: 4 September 2017 / Accepted: 19 March 2018 / Published online: 21 April 2018

(C) The Author(s) 2018

\begin{abstract}
We predict the sterile neutrino spectrum of some of the key solar nuclear reactions and discuss the possibility of these being observed by the next generation of solar neutrino experiments. By using an up-to-date standard solar model with good agreement with current helioseismology and solar neutrino flux data sets, we found that from solar neutrino fluxes arriving on Earth only 3-4\% correspond to the sterile neutrino. The most intense solar sources of sterile neutrinos are the $p p$ and ${ }^{7} \mathrm{Be}$ nuclear reactions with a total flux of $2.2 \times 10^{9}$ and $1.8 \times 10^{8} \mathrm{~cm}^{2} \mathrm{~s}^{-1}$, followed by the ${ }^{13} \mathrm{~N}$ and ${ }^{15} \mathrm{O}$ nuclear reactions with a total flux of $1.9 \times 10^{7}$ and $1.7 \times 10^{7} \mathrm{~cm}^{2} \mathrm{~s}^{-1}$. Moreover, we compute the sterile neutrino spectra of the nuclear proton-proton nuclear reactions $-p p$, hep and ${ }^{8} B$ and the carbon-nitrogen-oxygen $-{ }^{13} N$, ${ }^{15} \mathrm{O}$ and ${ }^{17} \mathrm{~F}$ and the spectral lines of ${ }^{7} \mathrm{Be}$.
\end{abstract}

\section{Introduction}

The standard neutrino flavour oscillation model with only three neutrinos has been very successful in explaining most of observational properties of neutrino fluxes. Nevertheless, the significant improvement on the experimental methods of neutrino detection during the past 15 years has revealed the existence of disagreement between the standard model of neutrino oscillations and experimental data. These discrepancies usually are referred to as neutrino anomalies. They have been reported in a number of different type of experiments: the Liquid Scintillator Neutrino Detector (LSND [2]), the Booster Neutrino Experiment, (MiniBooNE (first phase of BooNE) [3,4]), reactor experiments [39] and the gallium experiments [20]. Several generalisations of the standard neutrino flavour oscillation model have been proposed in the literature to explain these neutrino anomalies (e.g., $[19,36])$. Among other solutions the most successful one is the prediction of the existence of a new type of neutrinos [38], known

a e-mail: ilidio.lopes@tecnico.ulisboa.pt as sterile neutrinos. However, this experimental hint, which can be explained by the existence of sterile neutrinos, has been challenged by more recent measurements made by the NEOS Experiment [33], the Super-Kamiokande detector [1] and the IceCube Collaboration [24]. Although the parameter space that allows the sterile neutrinos to explain the neutrino anomalies has been significantly reduced, their existence can still be accommodated within the current set of observations.

Sterile neutrinos are very well-motivated elementary particles and can be obtained from a trivial extension of the standard model of particle physics. ${ }^{1}$ Their name reflects the fact that by definition sterile neutrinos are not affected by any of the known forces of nature except gravity, since these particles carry no charges and are singlets under all gauge groups of the standard model. This type of neutrinos, unlike other particle candidates, provides an unified description of three major problems in the framework of the standard model: the origin of the neutrino masses and the flavour oscillation model [32], the absence of primordial anti-matter in the Universe and the existence of dark matter [13]. Although many of the properties of this class of neutrinos are fixed by the generalised standard model, other properties of sterile neutrinos remain undefined, like their masses. Actually, all sorts of theoretical arguments have been proposed to explain the existence of sterile neutrinos with a very large range of mass scales: from neutrinos with very light masses much smaller than $\mathrm{eV}$ [22] up to very heavy neutrinos with masses of $10^{16}$ $\mathrm{GeV}$ [37]. In recent years, particular attention has been given to the sterile neutrino candidates with masses of a few $\mathrm{eV}$, hundred $\mathrm{keV}$ and a few $\mathrm{GeV}$ [42] as several experiments are already obtaining data or are under construction with the goal of probing the existence of sterile neutrinos on these mass ranges.

The exact number of sterile neutrinos is not known, but even the simplest of the neutrino flavour oscillation models

\footnotetext{
${ }^{1}$ In the remainder of the article, the standard model will always refer to the standard model of particle physics if not stated otherwise.
} 
in which only one extra sterile neutrino $v_{s}$ is added up to the standard active neutrinos $\left(v_{e}, v_{\tau}, v_{\mu}\right)$ is able to resolve many of the known experimental discrepancies - the neutrino anomalies. This model is the so-called $3+1$ neutrino flavour oscillation model in which the generalised mass matrix leads to the mixing of four neutrinos $\left(v_{e}, v_{\tau}, v_{\mu}, v_{s}\right)$. This basic model presents very compelling results compatible with experimental data $[12,40]$. Following the literature (e.g., [12]), in our study we will focus on the $3+1$ sterile neutrino in which two additional parameters are included to characterise the sterile neutrino flavour oscillations: the mass splitting $\left|\Delta m_{41}^{2}\right|$ and the mixing angle $\theta_{14}$.

The mass splitting and the mixing angle (as defined in the $3+1$ flavour neutrino oscillation model), which have been estimated by different research groups using several observational data sets and different fitting techniques, found very similar values for both quantities. A global analysis of the neutrino oscillation data in the $3+1$ parameter space made by Giunti et al. [21] found that $\Delta m_{41}^{2}$ could take a value between $0.82-2.19 \mathrm{eV}^{2}$ at $99.7 \%$ confidence level. Similarly, Ko et al. [33] making an independent analysis have obtained identical values for $\left|\Delta m_{41}^{2}\right|$, specifically mass values in the range $0.2-2.3 \mathrm{eV}^{2}$. The determination of $\theta_{14}$ by different research groups leads also to similar values. For instance, Billard et al. [12] and Ko et al. [33], found for the upper limit of $\theta_{14}$ the values $10.6^{\circ}(0.1855)$ and $9.2^{\circ}(0.1582)$. These values for $\theta_{14}$ differ only slightly from the first determination made by Palazzo [40] who found $11.7^{\circ}(0.204)$. Conveniently, for our calculations we choose for the fiducial value of $\theta_{14}$ the most recently determined value: $9.2^{\circ}(0.1582)$ or $\sin ^{2}\left(2 \theta_{14}\right)=0.1[33]$.

Since sterile neutrinos do not take part in the weak interaction, the most obvious way to observe their effect is via their mixing with the active neutrinos. Therefore, with the goal of determining the sterile neutrino properties, in this study we predict the spectra of sterile and electron neutrinos coming from the Sun, in the hope that this will contribute to their discovery by "direct" detection by one of the ongoing experiments, the ultimate silver bullet that will prove their existence. Specifically, we compute the sterile neutrino and electron neutrino spectra of some of the key nuclear reactions occurring in the Sun's core. These two types of neutrino spectra, as we will discuss in this work, results from the conversion of electron neutrinos into sterile neutrinos and from the survival of electron neutrinos, due to their oscillation between the four neutrino flavours, as these particles propagate through space (vacuum and matter). Specifically, we predict the shape of the different solar sterile and electron neutrino spectra of the key neutrino nuclear reactions of the proton-proton (PP) chain and carbon-nitrogen-oxygen (CNO) cycle occurring in the interior of the present Sun. The calculation will be made for the current solar standard model which used the most up-to-date physics (equation of state, opacities, nuclear reactions rates and microscopic diffusion of heavy elements). The details of the physics of the solar model used in this study can be found in Lopes and Silk [30], Lopes and Turck-Chièze [31]. This solar model predicts the sound speed profile and solar neutrino fluxes in agreement with observational data and similar to other models found in the literature (i.e., [41,43]).

In the next section, we discuss in some detail the $3+1$ sterile neutrino flavour oscillation model. In the following section, we present the spectra predictions for sterile and electron neutrinos of several of the key solar nuclear reactions. In the last section, we discuss the results and their implications for the next generation of sterile neutrino experiments.

\section{Neutrino flavour oscillation models}

This study focuses on the simple and popular $3+1$ neutrino flavour oscillation model with four neutrinos: $v_{e}, v_{\mu}, v_{\tau}$ and $v_{s}$. The addition of an extra (sterile) neutrino leads to a new flavour oscillation component on the $3+1$ neutrino model defined by a new mass splitting $\left|\Delta m_{41}^{2}\right|$ and a new mixing angle $\theta_{14}$. As discussed previously, a recent fit of the experimental data to the $3+1$ neutrino model done by Billard et al. [12] found $\sin ^{2} \theta_{14}=0.034$ (or $\theta_{14} \sim 10.6^{\circ}(0.1855)$ ), $\Delta m_{21}^{2}=(7.5 \pm 0.14) \times 10^{-5} \mathrm{eV}^{2}, \sin ^{2}\left(\theta_{12}\right)=0.300 \pm 0.016$ and $\sin ^{2} \theta_{13}=0.03$. In our calculations of the $3+1$ neutrino oscillation model we will use these parameters. However, the value of $\theta_{14}$ is replaced by a recent estimation of $9.2^{\circ}(0.1582)$ obtained by Ko et al. [33]. This choice of $\theta_{14}$ does not change our conclusions since the difference between the two values is small enough not to affect our results. It is important to highlight that the experimental data used by Billard et al. [12] for the previous fits comprises data from solar detectors like SNO, Super Super-Kamiokande, Borexino, Homestake, and Gallium experiments and reactors data from the very long baseline KamLAND. Effectively, the inclusion of more experimental data, like the data sets coming from short baseline detectors such as the DAYA-BAY, RENO, and Double Chooz reactor experiments changes only slightly the value of $\sin ^{2} \theta_{14}$ and once again its magnitude variation is too small to significantly affect our results and conclusions [12]. Actually, although $\sin ^{2} \theta_{14}$ has changed over the years, its present value is only slightly different from the value originally found by Palazzo [40]: $\sin ^{2} \theta_{14}=0.041$. Moreover, we observe that the $\left|\Delta m_{41}^{2}\right|$ determined by Ko et al. [33] to be in the mass range of $\sim 0.2$ to $2.3 \mathrm{eV}^{2}$ is much larger than the mass splittings, $\left|\Delta m_{21}^{2}\right|$ and $\left|\Delta m_{32}^{2}\right|$ estimated to be of the order of $10^{-5}$ and $10^{-3} \mathrm{eV}^{2}[17,33]$. We note that in the $3+1$ neutrino model discussed in our study only the mixing angle $\theta_{14}$ is considered to have a value different from zero $[12,40]$. We stress that this is a very good approximation, since a fit to the $3+1$ neutrino model (with more mix- 
ing angles), besides the new mixing angle $\theta_{14}$, has additional mixing angles like $\theta_{24}$ and $\theta_{34}$ (associated with other possible flavour oscillations), found relatively small values for $\theta_{24}$ and $\theta_{34}$ [34]. Therefore setting these last mixing angles to zero will not affect our results and conclusions. The experimental facts discussed above justify the analytical neutrino oscillation model that we will discuss in the next subsection.

Finally, we point out that our $3+1$ neutrino model has parameters (excluding $\Delta m_{41}$ and $\theta_{41}$ ) very similar to the ones found for the standard three neutrino flavour oscillation model. For reference, a recent estimation of parameters made by Gonzalez-Garcia et al. [17] using the standard threeflavour neutrino oscillation model to fit the combined experimental data set obtained from atmospheric experiments, solar detectors and particle accelerators found the following set of parameters: $\Delta m_{31}^{2} \sim 2.457 \pm 0.04510^{-3} \mathrm{eV}^{2}$ (or $\left.-2.449 \pm 0.04810^{-3} \mathrm{eV}^{2}\right), \Delta m_{21}^{2} \sim 7.500 \pm 0.01910^{-5} \mathrm{eV}^{2}$ $\sin ^{2} \theta_{12}=0.304 \pm 0.013, \sin ^{2} \theta_{13}=0.0218 \pm 0.001, \sin ^{2} \theta_{23}$ $=0.562 \pm 0.032$ and $\delta_{C P}=2 \pi / 25 n$ with $n=1, \ldots, 25$. This set of parameters of values is also confirmed by other authors $[9,18]$.

\subsection{The standard and $3+1$ neutrino flavour models}

The neutrino oscillation model with a fourth sterile neutrino $v_{s}$ is such that the neutrino flavours $\left(v_{e}, v_{\mu}, v_{\tau}, v_{s}\right)$ and the mass eigenstates $\left(v_{1}, v_{2}, v_{3}, v_{4}\right)$ are connected through a $4 \times 4$ unitary mixing matrix $U$. We choose for $U$ the parametrisation proposed by Palazzo [40] which is optimised to study the solar neutrino sector. It is worth highlighting that this peculiar parametrisation is somehow between an admixture of flavour eigenstates and matter eigenstates, as discussed by Cirelli et al. [14]. Such a $U$ formulation ensures that this $3+1$ neutrino model has the required sensitivity to the flavourtype and mass-type admixtures needed to study the solar data sector. In particular, this $3+1$ mixing matrix is such that the standard neutrino model corresponds to $\theta_{14}=0$. A detailed account of the properties of this model can be found in Palazzo [40]. In particular, the electron neutrino $v_{e}$ oscillation with the $\left(v_{1}, v_{2}\right)$ mass eigenstates is only slightly affected by the decoupled $\left(v_{3}, v_{4}\right)$ mass eigenstates for which the associated mixing angles are very small. In such a neutrino model, the MSW effect is computed in the hierarchical limit $\Delta m_{21}^{2} / 2 E \ll \Delta m_{31}^{2} / 2 E \ll \Delta m_{41}^{2} / 2 E$ where $E$ is the energy of the neutrino, an $\Delta m_{i 1}^{2}=m_{i}^{2}-m_{1}^{2}$ where $m_{i}$ is the mass of each neutrino. This $3+1$ neutrino model is an obvious generalisation of the standard (three active) neutrino model [35]. In both cases neutrino oscillations are approximated by an effective two neutrino oscillation model. As a consequence the $v_{3}$ and $v_{4}$ evolve independently from each other and completely independent from the doublet, $\left(v_{1}, v_{2}\right)$.
2.2 The eigenvalues of the mass matrix in $3+1$ model

In the Sun, as the experimental data on neutrino fluxes is limited, it is sufficient to represent the survival or conversion of neutrino flavour, using a two neutrino flavour oscillation model [35]. The neutrinos emitted by the solar nuclear reactions are typically of low energy, since these neutrinos have always an energy smaller than $20 \mathrm{MeV}$. Accordingly, some of the properties of solar neutrinos can be well understood by means of a two active neutrino flavour model: one electron neutrino $v_{e}$ and a second active neutrino, which we choose to be $v_{\mu}$, since the $v_{\tau}$ mixing angle is very small. Another way to interpret this approximation is to assume that $v_{\mu}$ now means the generic active $v_{\mu \tau}$, if not stated otherwise. In this much simpler 2 (active) +1 (sterile) neutrino flavour model $\left(v_{e}, v_{\mu}, v_{s}\right)$, the properties of the sterile neutrino $v_{s}$ can be computed as an approximation to the $\left(v_{e}, v_{\mu}, v_{\tau}, v_{s}\right)$ neutrino model. Accordingly, this $2+1$ neutrino model has the active mass eigenstates $v_{1}$ and $v_{2}$, as well as a sterile mass eigenstate ${ }^{2} v_{4}[14,22]$. The $2+1$ flavour model in the absence of sterile neutrinos has the following eigenvalue expressions [22]. If $\lambda_{i}(i=1,2)$ are the correspondent eigenvalues of the mass matrix, they read

$\lambda_{i}=\frac{M^{2}}{4 E}+\frac{V_{e}+V_{n}}{2} \mp \sqrt{\left(\Delta_{c}-\frac{V_{e}-V_{n}}{2}\right)^{2}+\Delta_{s}^{2}}$,

where $M^{2}=m_{1}^{2}+m_{2}^{2}, \Delta c \equiv \Delta m_{21}^{2} \cos \left(2 \theta_{21}\right) /(4 E)$ and $\Delta s \equiv \Delta m_{21}^{2} \sin \left(2 \theta_{12}\right) /(4 E), V_{e}=\sqrt{2} G_{F}\left(n_{e}-n_{n} / 2\right)$ and $V_{n}=-\sqrt{2} G_{F} n_{n} / 2$, where $G_{F}$ is the Fermi constant and $n_{e}(r)$ and $n_{n}(r)$ are the profile of electron and neutron densities of the solar interior. The electron density $n_{e}(r)$ is given by $N_{o} \rho(r) / \mu_{e}(r)$, where $\mu_{e}(r)$ is the mean molecular weight per electron, $\rho(r)$ is the density of matter in the solar interior, and $N_{o}$ is Avogadro's number. The density of neutrons $n_{n}(r)$ is computed in a manner similar to $n_{e}(r)$ where the mean molecular weight per electron $\mu_{e}(r)$ is replaced by the mean molecular weight per neutron $\mu_{n}(r)$.

Figure 1 shows the current abundances of electrons and neutrons inside the Sun (as a function of the solar radius) for its present age as predicted by an up-to-date standard solar model [30]. The large value of $n_{n}(r)$ in the centre of the Sun results from the fact that the Sun's core is made of more than $70 \%$ of ${ }^{4} \mathrm{He}$. This occurs as a result of this element being continuously produced (nucleosynthesis) by the conversion of four protons (zero neutrons) into an ion of ${ }^{4} \mathrm{He}$ (two neutrons). This process has being occurring since our star arrived to the main sequence 4.5 Gyr ago and will continue to do it for the next 5 Gyr (until the Sun leaves the main sequence). As a consequence, at the present age

\footnotetext{
2 The option to use the subscript 4 for the mass eigenstate of the sterile neutrino is motivated by the fact of maintaining a simple correspondence between the $2+1$ and $3+1$ neutrino flavour models.
} 


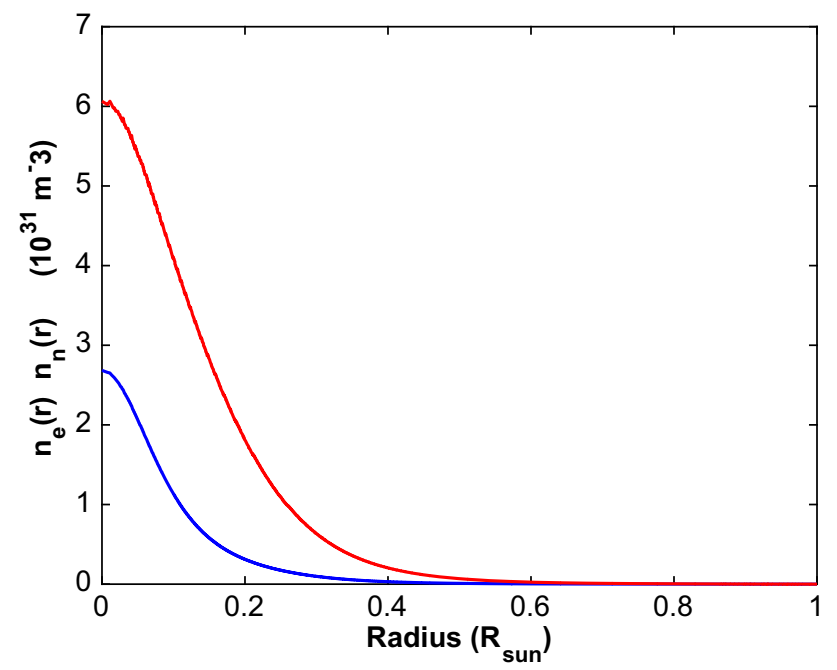

Fig. 1 The plasma of the Sun's core is made of ions and free electrons. The plot shows the variation of the number density of electrons $n_{e}(r)$ (red curve) and neutrons $n_{n}(r)$ (blue curve). In the computation of this quantities we used the solar standard model which is in excellent agreement with the helioseismic data. See Lopes and Silk [30] for details

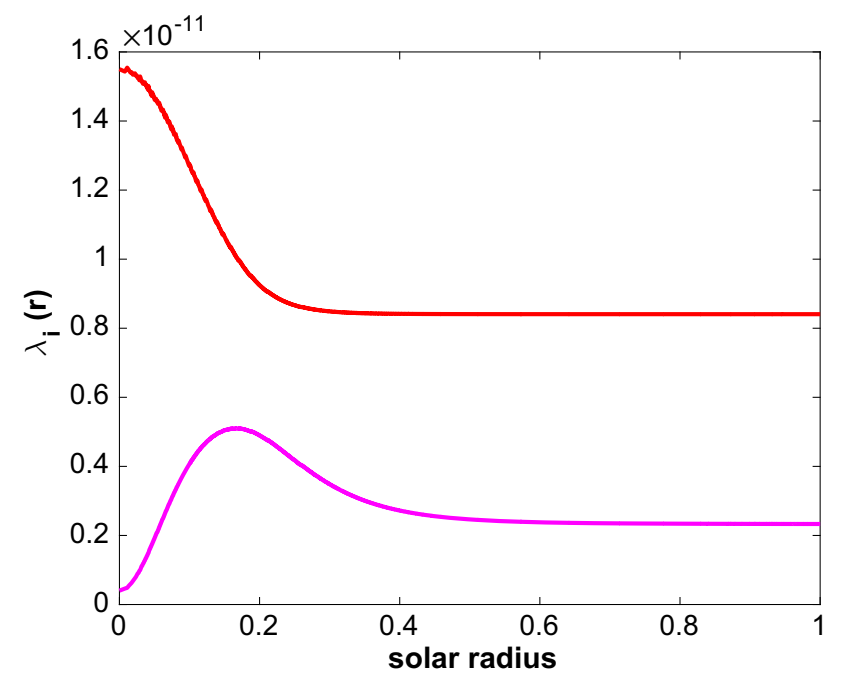

Fig. 2 The level crossing scheme: The mass eigenvalues $\lambda_{1}$ (red curve) and $\lambda_{2}$ (magenta curve) as a function of the solar radius (cf. Eq. (1)). These $\lambda$ values correspond to a neutrino with an energy $E=3.5 \mathrm{MeV}$

the core of the Sun has a larger amount of neutrons than the more external layers, leading to a decrease of $n_{n}(r)$ from the centre towards the surface of the star. This important point is illustrated in Fig. 1 where we show for electrons and neutrons the relative density and mean molecular weight per particle inside the Sun.

For illustrative purposes we assume that $m_{i}^{2} \approx \Delta m_{i 1}^{2}$ (with $i \neq 1$ ). Specifically, $m_{1}^{2} \approx 0, m_{2}^{2} \approx 7.5 \times 10^{-5} \mathrm{eV}^{2}$ (with $\theta_{12} \approx 0.6$ ), $m_{3}^{2} \approx 2.4 \times 10^{-3} \mathrm{eV}^{2}$ (with $\theta_{13} \approx 0.13$ ), and $m_{4}^{2} \approx 1.0 \mathrm{eV}^{2}$ (with $\theta_{14} \approx 0.16$ ). The $\lambda_{i}(i=1,2)$ can be seen in Fig. 2. In this model, for which $m_{1}<m_{2}<m_{3}<m_{4}$,

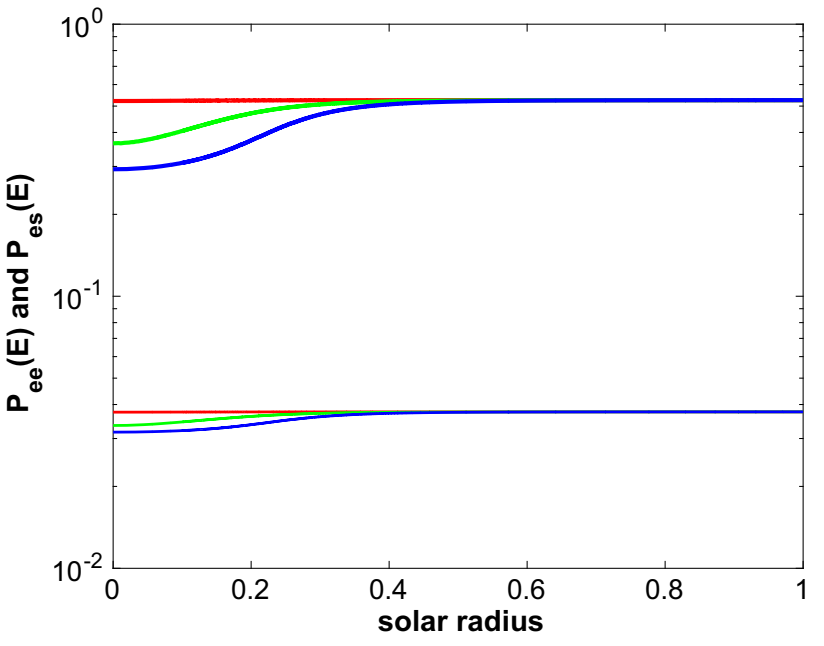

Fig. 3 The neutrino probability functions: $P_{e s}(E)$ corresponds to the probability of conversion of $v_{e}$ to $v_{s}$ neutrinos given by Eq. (2) (bottom set of curves); and $P_{e e}(E)$ corresponds to the survival probability of $v_{e}$ given by Eq. (3) (top set of curves). The red, green and blue curves correspond to neutrinos with energy of $0.1,5$ and $15 \mathrm{MeV}$, which correspond to typical values of neutrino energies produced inside the Sun's core. In the computation of these quantities we used the standard solar model (see Lopes and Silk [30] for details)

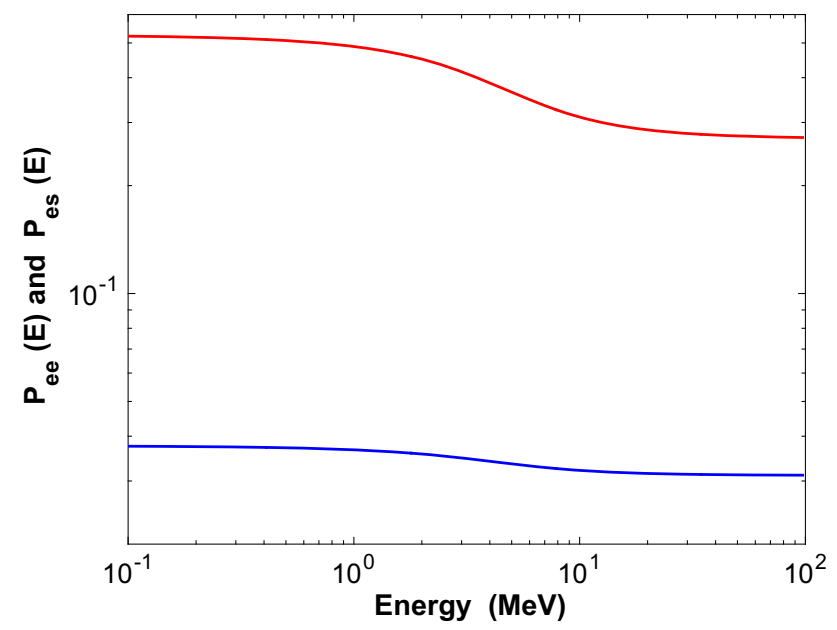

Fig. 4 The neutrino probability functions as a function of the neutrino energy: $P_{e s}(E)$ corresponds to the probability of conversion of $v_{e}$ to $v_{s}$ neutrinos (blue curve); $P_{e e}(E)$ corresponds to the survival probability of $v_{e}$ neutrinos (red curve). See Fig. 3 for details

there is only a relevant resonance in the system associated with 1-2 level crossing. The 1-2 resonance energy is given by the expression $E_{12}=\Delta m_{21}^{2} \cos \left(2 \theta_{12}\right) /\left(2 \sqrt{2} G_{F} n_{e}\right)$. As the electron neutrino moves outwards, the electronic density decreases and the neutrino eventually go through the resonance region $E_{12}(r)$ and beyond. However, since the electron density $n_{e}(r)$ in the Sun's core varies slowly, i.e., $\mathrm{d} n_{e}(r) / \mathrm{d} r$ is small, the propagation is adiabatic, the neutrino state will remain in the same mass eigenstate, and then the $v_{e}$ will emerge associated with $v_{1}$. The flavour composition of $v_{2}$ is 


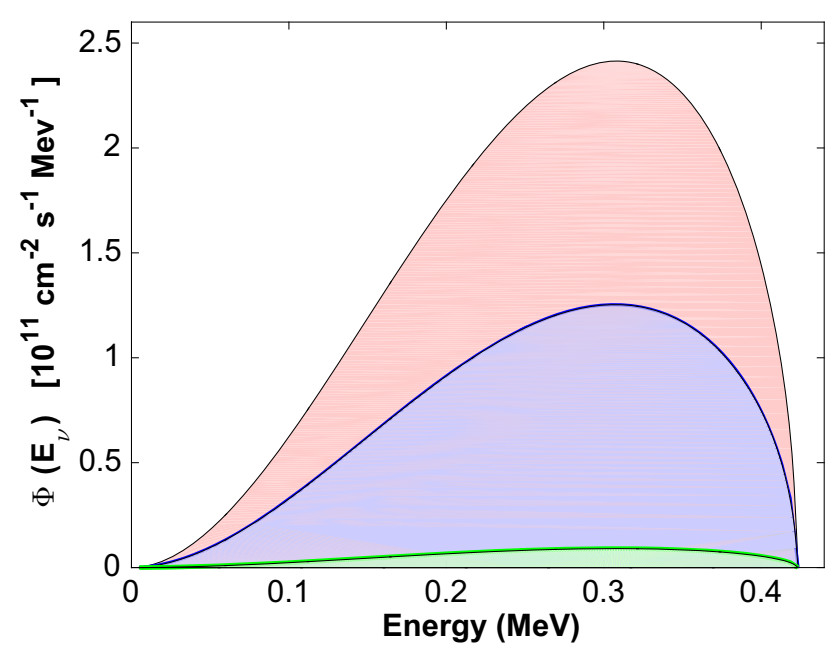

Fig. 5 Neutrino spectrum of $p p$ nuclear reaction: the $\Phi_{t}(E)$ (red area) is the electron neutrino spectrum inside the Sun. $\Phi_{e e}^{\odot}(E)$ (blue area) and $\Phi_{e s}^{\odot}(E)$ (green area) are the survival electron and sterile solar neutrino spectra for the PP neutrinos $3+1$ neutrino flavour oscillation model

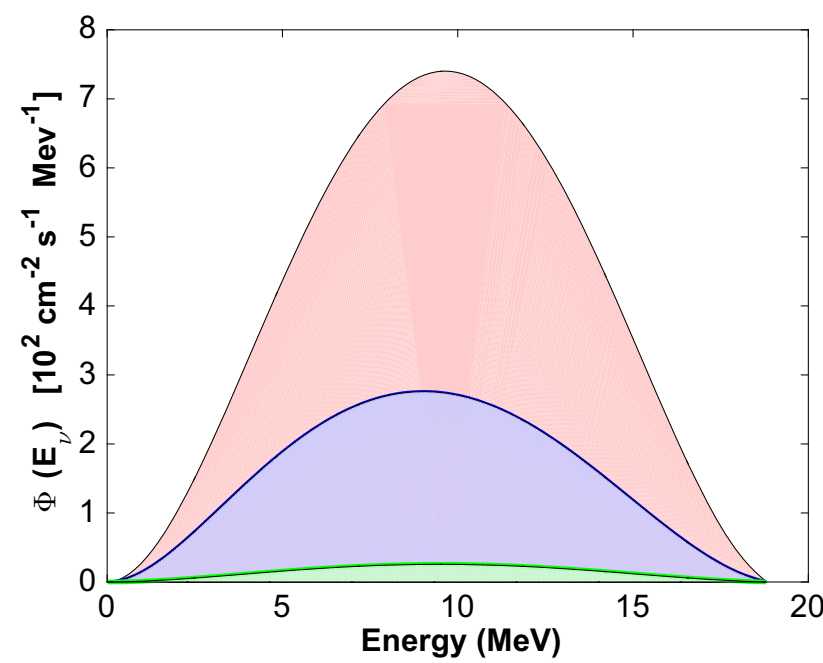

Fig. 6 Neutrino spectrum of hep nuclear reaction. The colour scheme is the same used in Fig. 5

now determined by the mixing angle in the vacuum and $\nu_{2}$ is now mostly $v_{\mu \tau}$.

We notice that for other neutrino models the eigenvalues can have additional resonances. For instance, de Holanda and Smirnov [22] have shown that, for a $2+1$ neutrino flavour model $\left(v_{e}, v_{\mu \tau}, v_{s}\right)$ with $m_{1}<m_{4}<m_{2}$, the sterile neutrino level $\lambda_{4}$ crosses $\lambda_{1}$. In this model, the $\lambda_{2}$ essentially decouples; therefore, it is not affected by the s-mixing. However, in such a neutrino model, due to the variation of the $n_{e}(r)$ in the Sun's core, the eigenvalues have two resonances: one related with the active neutrinos, the 1-2, i.e., the same resonance mentioned previously; and a new resonance, the 1-4, which corresponds to $\lambda_{4}$ crossing $\lambda_{1}$ in a region at the right

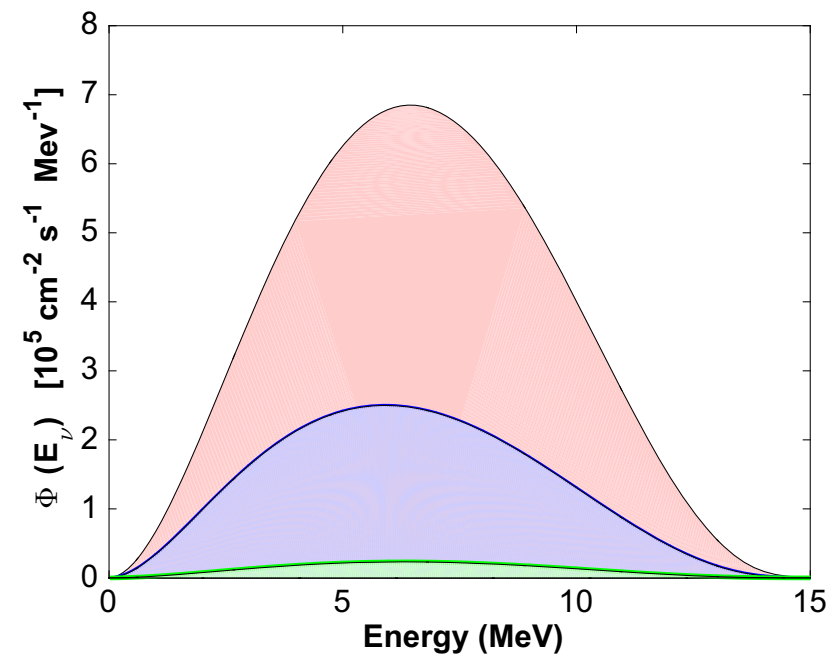

Fig. 7 Neutrino spectrum of ${ }^{8} B$ nuclear reaction. The colour scheme is the same used in Fig. 5
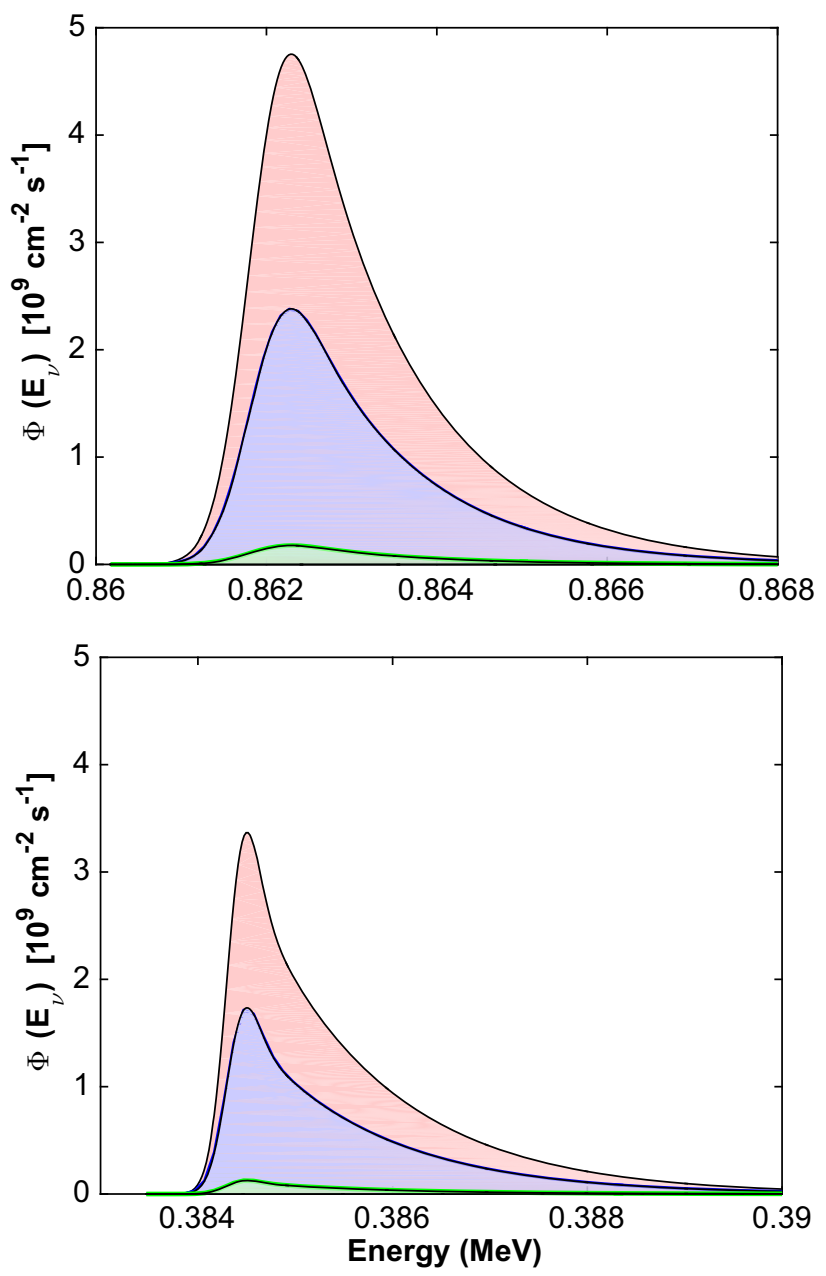

Fig. 8 Neutrino of the two line spectra of ${ }^{7} \mathrm{Be}$ nuclear reaction. The colour scheme is the same used in Fig. 5 
of the 1-2 resonance. ${ }^{3}$ However, this case does not occur in our study, since in our $2+1$ model $\lambda_{4}$ is much larger than $\lambda_{1}$ and $\lambda_{2}$ and consequently does not cross any of them.

\subsection{The conversion of sterile neutrino in electronic neutrinos}

The goal of this work is to predict the spectra of sterile and electronic neutrinos emitted by the Sun for which their specific shapes strongly depend on the properties of the plasma of the solar interior. As such, in the following we start to compute the probabilities of conversion of electron neutrino to sterile neutrino $P_{e s}\left[\equiv P\left(v_{e} \rightarrow v_{s}\right]\right.$ arriving on Earth. Equally we also compute the survival probability of electron neutrino $P_{e e}\left[\equiv P\left(v_{e} \rightarrow v_{e}\right]\right.$. In this $3+1$ neutrino flavour oscillation model like in the standard three-flavour oscillations (e.g., [11]), the neutrino oscillates between the available flavours due to oscillations in vacuum and matter (MikheyevSmirnov-Wolfenstein (MSW), e.g., [47]). The survival and conversion probabilities of a four-flavour neutrino oscillation can be reduced to a modified three-flavour and two-flavour neutrino oscillation model. These simplifications result from the difference of magnitude between mass splittings and mixing angles. A detailed discussion as regards the neutrino flavour oscillation model can be found in Gonzalez-Garcia and Maltoni [19].

Since the evolution of neutrinos in matter is adiabatic, their contribution for $P_{e s}$ and $P_{e e}$ can be cast in the form of simple expressions [40]. Following the usual parametrisation of the neutrino mixing matrix this leads to the following expression for $P_{e s}$ (probability conversion from $v_{e}$ to $v_{s}$ ):

$P_{e s}=s_{14}^{2} c_{14}^{2} c_{13}^{4} \bar{P}_{e e}^{2 v}+s_{14}^{2} c_{14}^{2} s_{13}^{4}+s_{14}^{2} c_{14}^{2}$,

where $c_{i j}=\cos \theta_{i j}$ and $s_{i j}=\sin \theta_{i j}$. Equally the survival probability of electron neutrinos $P_{e e}$ reads

$P_{e e}=c_{14}^{4} c_{13}^{4} \bar{P}_{e e}^{2 v}+c_{14}^{4} s_{13}^{4}+s_{14}^{4}$.

In the previous equations $\bar{P}_{e e}^{2 v}$ is the electron neutrino survival probability in a two-flavour neutrino oscillation model. It reads

$\bar{P}_{e e}^{2 v}=c_{12}^{2}\left(c_{12}^{m}\right)^{2}+s_{12}^{2}\left(s_{12}^{m}\right)^{2}$,

where $\theta_{12}^{m}$ is the matter angle that depends equally of the fundamental parameters of neutrino flavour oscillations and the properties of solar plasma. $\theta_{12}^{m}$ can be equally determined from one of the following expressions:

$$
\cos \left(2 \theta_{12}^{m}\right)=\frac{\cos \left(2 \theta_{12}\right)-V_{m}(r)}{\sqrt{\left[\cos \left(2 \theta_{12}\right)-V_{m}(r)\right]^{2}+\left[\sin \left(2 \theta_{12}\right)\right]^{2}}},
$$

\footnotetext{
3 As explained by de Holanda and Smirnov [22], in principle $\lambda_{4}$ could cross $\lambda_{1}$ twice: on the one hand above and on the other hand below the $1-2$ resonance.
}

or

$$
\sin \left(2 \theta_{12}^{m}\right)=\frac{\sin \left(2 \theta_{12}\right)}{\sqrt{\left[\cos \left(2 \theta_{12}\right)-V_{m}(r)\right]^{2}+\left[\sin \left(2 \theta_{12}\right)\right]^{2}}} .
$$

In the previous expressions $V_{m}(r)$ is the effective matter potential given by

$V_{m}(r)=c_{13}^{2}\left(c_{14}^{2}+f_{m}(r) s_{14}^{2}\right) \frac{2 E \sqrt{2} G_{F} n_{e}(r)}{\Delta m_{21}^{2}}$.

The factor $f_{m}(r)$ in a function, which gives the ratio of the neutron density $n_{n}(r)$ in relation to the electron density $n_{e}(r)$ at each radius of the solar interior, reads

$f_{m}(r)=\frac{1}{2} \frac{n_{n}(r)}{n_{e}(r)}$

The variation of $V_{m}(r)$ (cf. Eq. (7)) depends on the radial variation of neutrons as well as electrons inside the star. We notice that in the particular case that $\theta_{14}=0$, the $3+1$ neutrino oscillation model becomes identical to the standard three neutrino oscillation model (for oscillations in vacuum and matter). Consequently, in this case $V_{m}(r)$ is reduced to a much simpler expression [11]: $V_{m}(r)=$ $c_{13}^{2} 2 E \sqrt{2} G_{F} n_{e}(r) / \Delta m_{21}^{2}$. These quantities (cf. Eq. (7)) have a major impact on the formation of the sterile and electronic neutrino spectra. Figures 3 and 4 show the $P_{e e}$ and $P_{e s}$ for the current standard solar model.

\subsection{Emission of electron neutrino spectra from solar nuclear reactions}

Since neutrinos are produced by nuclear reactions (either the PP chains or the CNO cycle) in different locations of the Sun's core, consequently, the survival probability of electron neutrinos will depend on the distance of the nuclear reaction to the centre of the Sun $[28,29]$. This will also affect indirectly the probability of conversion of electron neutrinos into sterile neutrinos. The average probability $P_{e e, k}$ of electron neutrinos of energy $E$ produced by a $k$ nuclear reaction region is given by

$P_{e e, k}(E)=A_{k}^{-1} \int_{0}^{R_{\odot}} P_{e e}(E, r) \phi_{k}(r) 4 \pi \rho(r) r^{2} \mathrm{~d} r$,

where $A_{k}$ is a normalisation constant that is equal to $\int_{0}^{R_{\odot}} \phi_{k}(r) 4 \pi \rho(r) r^{2} \mathrm{~d} r . \phi_{k}(r)$ is the electron neutrino emission function for the following $k$ nuclear reactions: $p p$, hep, ${ }^{8} \mathrm{~B},{ }^{7} \mathrm{Be},{ }^{13} \mathrm{~N},{ }^{15} \mathrm{O}$ and ${ }^{17} \mathrm{~F}$. In this study, we consider that all neutrinos produced in the solar nuclear reactions are of electron flavour as predicted by standard nuclear physics; therefore, the local density of neutrons only affects the $P_{e e, k}(E)$ by affecting the flavour of electron neutrinos due to the existence of a new sterile neutrino $v_{s}$. It is important to note that $\phi_{k}(r)$, the neutrino source of each nuclear reaction, is 

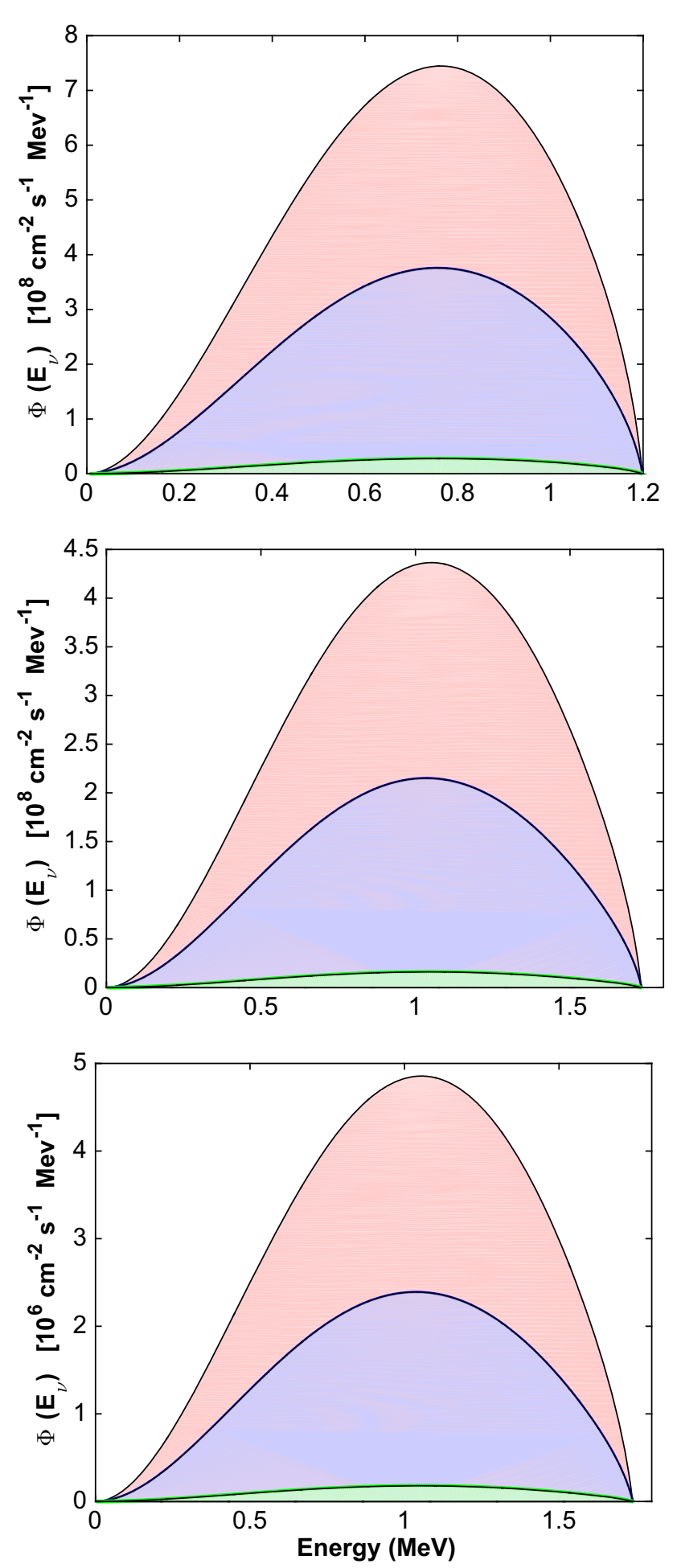

Fig. 9 The solar neutrino spectra for $\mathrm{CNO}$ neutrinos: Neutrino spectra of the cno nuclear reactions. $N 13 v$ (top), $O 15 v$ (middle) and $F 17 v$ (bottom). The colour scheme is the same as used in Fig. 5

sensitive to the local values (radius $r$ ) of the temperature, molecular weight, density, neutron and electronic densities.

In a $3+1$ neutrino flavour oscillation model, as neutrinos propagate in the Sun's interior, the neutrino flavours oscil- late between the four flavour states $v_{e}, v_{\mu}, v_{\tau}$ and $v_{s}$ either due to vacuum or to matter oscillations. Although in outer space (between the Sun and the Earth) the flavour oscillations due to matter effects are expected to be small, the high density of the solar interior medium will enhance the scattering of neutrinos with other elementary particles, like electrons and neutrons, leading to the increase of the probability of the neutrino oscillating between different flavour states. The particular distribution of electrons and neutrons in the solar interior leads to characteristic shapes of neutrino spectra (of electron neutrinos and sterile neutrinos) for each of the neutrino nuclear reactions occurring in the Sun's interior, as we will discuss in the next section (see Figs. 5, 6, 7, 8, 9).

\section{Prediction of the solar sterile and electron neutrino spectra}

The spectra of electron neutrinos from any specific nuclear reaction, like the $\beta$-decay processes occurring in the Sun's core, are known to be essentially independent of the properties of the solar plasma. Actually, the neutrino spectra emitted by these nuclear reactions occurring inside the Sun are identical to the same spectra emitted by the seminar nuclear reactions tested on many laboratories on Earth. Therefore, it is reasonable to assume that the neutrino emission spectra are the same in the two cases [28]. Since the $\beta$-decay processes only produce electron neutrinos, using the theory of neutrino flavour oscillation, it is possible to predict in detail the spectrum shapes of sterile and electronic neutrinos arriving on Earth.

Here we make predictions of the sterile neutrino spectra of the main nuclear reactions occurring in the Sun, in the hope that this can be used as a guide for the future generation of neutrino detectors, which could help to establish (or rule out) the existence of sterile neutrinos. Once that the neutrinos emitted in the solar nuclear reactions are all of electron flavour, $v_{e}$, the existence of other neutrino flavours in the solar neutrino spectrum (as it is possible to observe on solar neutrino detectors) is uniquely related with the nature of neutrino flavour oscillations, which leads to the convection of $v_{e}$ on other neutrino flavours: $v_{\mu}, v_{\tau}$ and $v_{s}$. Accordingly, the electron neutrino spectrum of the $k$ nuclear reaction is defined as $\Phi_{k, t}(E)$ (with the subscript $t$ referring to the emission electron neutrino spectrum of the nuclear reaction $k$ ), and $\Phi_{k, s \odot}(E)$ and $\Phi_{k, e \odot}(E)$ are the sterile neutrino spectrum and the electron neutrino spectrum arriving on Earth. The function $\Phi_{k, t}(E)$ corresponds to the electron neutrino spectrum of a specific nuclear reaction computed theoretically, and in many cases measured in the laboratory. The ${ }^{8} \mathrm{~B}$ neutrino spectrum is a very good example. The ${ }^{8} \mathrm{~B}$ neutrino spectrum [6] has been shown to fit the experimental data with a high degree of accuracy (e.g., [46]). Hence, the sterile neutrino spectrum 
Table 1 Solar neutrino sources of sterile neutrinos

\begin{tabular}{llll}
\hline Neut. source & $\Phi_{t}^{\mathrm{a}}, \mathrm{cm}^{-2} \mathrm{~s}^{-1}$ & $\Phi_{e}, \mathrm{~cm}^{-2} \mathrm{~s}^{-1}$ & $\Phi_{s}, \mathrm{~cm}^{-2} \mathrm{~s}^{-1}$ \\
\hline pp chain & & \\
$\quad$ pp & $5.94 \times 10^{10}$ & $3.09 \times 10^{10}(52 \%)$ & $2.22 \times 10^{9}(3.7 \%)$ \\
$\quad$ hep & $7.88 \times 10^{3}$ & $2.88 \times 10^{3}(37 \%)$ & $2.64 \times 10^{2}(3.4 \%)$ \\
${ }^{8} \mathrm{~B}$ & $5.27 \times 10^{6}$ & $1.91 \times 10^{6}(36 \%)$ & $1.76 \times 10^{5}(3.3 \%)$ \\
${ }^{7} \mathrm{Be} 861.3 \mathrm{keV}$ & $4.75 \times 10^{9}$ & $2.38 \times 10^{9}(50 \%)$ & $1.76 \times 10^{8}(3.7 \%)$ \\
${ }^{7} \mathrm{Be} 384.3 \mathrm{keV}$ & $4.75 \times 10^{9}$ & $2.44 \times 10^{9}(51 \%)$ & $1.77 \times 10^{8}(3.7 \%)$ \\
${ }_{\mathrm{cno} \mathrm{cycle}}$ & & & \\
${ }^{13} \mathrm{~N}$ & $5.32 \times 10^{8}$ & $2.69 \times 10^{8}(51 \%)$ & $1.97 \times 10^{7}(3.7 \%)$ \\
${ }^{15} \mathrm{O}$ & $4.49 \times 10^{8}$ & $2.21 \times 10^{8}(49 \%)$ & $1.65 \times 10^{7}(3.7 \%)$ \\
${ }^{17} \mathrm{~F}$ & $5.01 \times 10^{6}$ & $2.47 \times 10^{6}(49 \%)$ & $1.84 \times 10^{5}(3.7 \%)$ \\
\hline
\end{tabular}

a The electronic neutrino fluxes predicted for the current solar model as in reference Lopes and Silk [30]

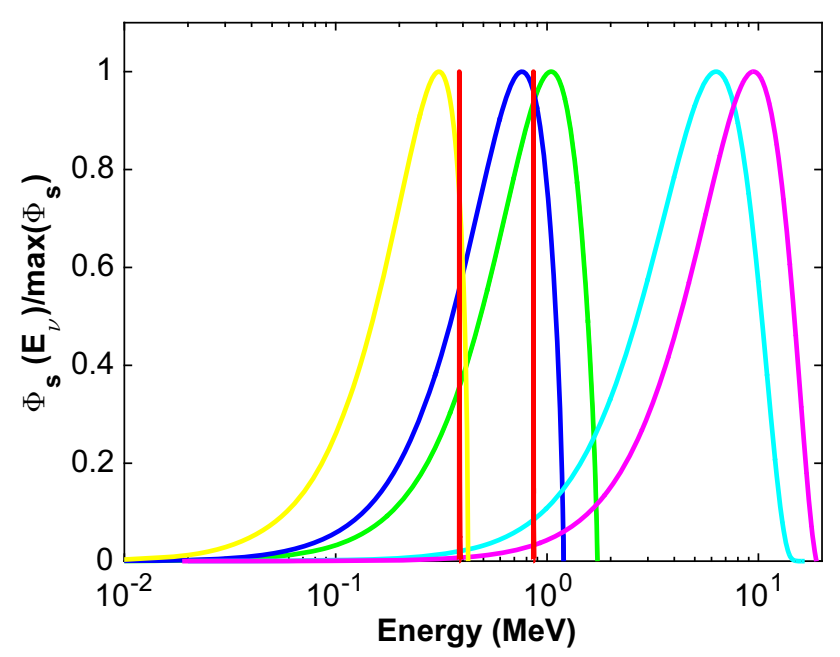

Fig. 10 Normalised sterile neutrino spectra for the Sun: $p p$ (yellow curve), ${ }^{8} B$ (cyan curve), Be7 (red curve) hep (magenta curve) $N 13$ (blue curve), $O 15$ and $F 17$ (green curve)

$\Phi_{k, s \odot}(E)$ is computed as

$\Phi_{k, s \odot}(E)=P_{e s, k}(E) \Phi_{k, t}(E)$,

where $k$ is equal to one of the following values: $p p$, hep, ${ }^{8} \mathrm{~B},{ }^{7} \mathrm{Be},{ }^{13} \mathrm{~N},{ }^{15} \mathrm{O}$ and ${ }^{17} \mathrm{~F}$. Similarly the electron neutrino spectrum $\Phi_{k, e \odot}(E)$ is given by

$\Phi_{k, e \odot}(E)=P_{e e, k}(E) \Phi_{k, t}(E)$.

Figures 5, 6, 7, 8 and 9 show the conversion of the emission neutrino spectra $\Phi_{k, t \odot}(E)$ in the two other spectrum flavours $\Phi_{k, e \odot}(E)$ and $\Phi_{k, s \odot}(E)$. Moreover, Table 1 shows the total neutrino fluxes computed for the present-day standard solar model [30]. For each nuclear reaction, $\Phi_{t}$ is the total neutrino flux emitted by the nuclear reaction, and $\Phi_{e}$ and $\Phi_{s}$ correspond to the flux fractions of $\Phi_{t}$ that arrive at the solar detector as electron neutrinos or sterile neutrinos.

We start to notice that due to neutrino oscillations the electron neutrino spectra arriving on Earth detectors for all the

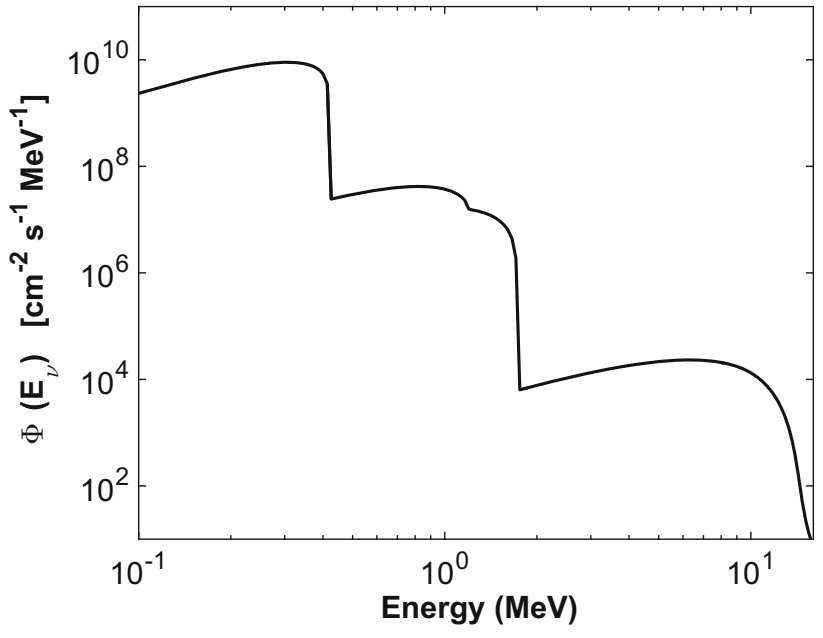

Fig. 11 The total sterile neutrino energy spectrum predicted by our standard solar model. The individual solar neutrino fluxes are shown in Table 1

solar nuclear reactions (considered in this study) are very different from the neutrino spectra emitted by the solar nuclear reactions (without neutrino oscillations). In particular, it is possible that the next generation of solar neutrino detectors will be able to measure $\Phi_{k, e \odot}(E)$. The sterile neutrino spectrum of all nuclear reactions have very small amplitudes and have identical spectral shapes although occurring at different neutrino energy ranges. In Table 1 we show the predicted neutrino fluxes for the leading solar nuclear reactions for the current solar standard model as well as the predicted ratios of sterile neutrino fluxes and electronic neutrino fluxes. The small values of sterile neutrinos are due to the very small mixing angle $\theta_{14}$. Only $4 \%$ of neutrinos arriving on Earth have a sterile flavour. This value is almost constant across all solar nuclear reactions. Nevertheless there is a small increase for the sterile neutrinos from the $p p$ reaction and a small decrease for sterile neutrinos from the ${ }^{8} B$ reaction. This is a consequence of the fact that the $p p$ and ${ }^{8} B$ nuclear reac- 
tions produce neutrinos with the smallest and the largest energies, which is due to the specific dependence sterile neutrino flavour oscillations with the local distribution of electrons and neutrons in the solar core leading to these specific variations. It is interesting to note that all sterile neutrinos in the solar neutrino spectrum have unique shapes related with the specific solar nuclear reactions on its origin. For instance, the two spectral lines of ${ }^{7} \mathrm{Be}$ have strong asymmetry in their shape, which could be helpful to look for hints related with sterile neutrinos in the solar neutrino spectrum. The strongest source of solar sterile neutrinos are the $p p$ and ${ }^{7} B e$ nuclear reactions with a total flux of $2.22 \times 10^{9}$ and $1.76 \times 10^{8} \mathrm{~cm}^{2} \mathrm{~s}^{-1}$, followed by the ${ }^{13} \mathrm{~N}$ and ${ }^{15} \mathrm{O}$ nuclear reactions with a total flux of $1.97 \times 10^{7}$ and $1.65 \times 10^{7} \mathrm{~cm}^{2} \mathrm{~s}^{-1}$ (cf. Table 1). Figure 11 shows the cumulative sterile neutrino spectrum coming from the Sun. The maximum emission of sterile solar neutrinos corresponds to an energy of $0.307 \mathrm{MeV}$ and a neutrino flux $9.0 \times 10^{9} \mathrm{~cm}^{2} \mathrm{~s}^{-1}$.

\section{Conclusion}

Sterile neutrinos are among the best candidates to resolve some of the leading problems in particle physics. At the same time these are very viable candidates for dark matter. Nevertheless, the hint of its existence has been questioned by a recent group of new experiments. The final proof beyond doubt of the existence of a sterile neutrino will pass by its direct detection.

In this study we have computed for the first time the sterile spectrum of several key solar nuclear reactions. We found that from the solar neutrino flux arriving on Earth only 3-4\% corresponds to sterile neutrinos. As expected, the strongest source is the $p p$ nuclear reaction with a total flux of $2.22 \times$ $10^{9} \mathrm{~cm}^{2} \mathrm{~s}^{-1}$ followed by the ${ }^{7} \mathrm{Be}$ nuclear reaction with a total flux one order of magnitude smaller. Equally, we have also predicted in detail the spectral shape of the sterile neutrino spectrum associated to the different solar nuclear reactions. In particular as shown in Fig. 10 the shape of these sterile neutrino spectra are quite distinct (Fig. 11).

A final point we should mention is that the Sun produces a significant amount of sterile neutrinos in the spectral range from $10 \mathrm{KeV}$ to $10 \mathrm{MeV}$, mostly coming for the $p p$ nuclear reaction (in the neutrino energy range from 0.01 up to $0.4 \mathrm{MeV}$ ), and ${ }^{7} \mathrm{Be}$ (spectral lines 0.861 and $0.384 \mathrm{MeV}$ ) that can affect experiments which are looking for relic (non-relativist) active and sterile neutrinos like the KATRIN and PTOLEMY Collaborations $[8,10]$. Indeed several experiments based on different principles have been proposed to detect such relic neutrinos (both components active and sterile neutrinos) $[5,15]$ : electron capture of decaying nuclei $[25,26]$, annihilation of high-energy cosmic neutrinos at the Z-resonance, atomic de-excitation [7,45] and neutrino capture using radioactive beta-decaying nuclei [44]. The latter seems to be the most promising technique with a few experiments already planned to that end like the KATRIN and PTOLEMY projects $[7,10,16,23,24]$. In a typical $\beta$-decay process like the one expected to be found in the PTOLEMY experiment, the relic neutrinos (cosmic neutrino background) will be shown as two independent kinks in the continuous $\beta$ spectrum, one due to the active neutrinos and a second small peak at higher energy associated to the admixture of sterile neutrinos [27]. The exact location of the energy peaks on the spectrum will depend on the masses of the active and sterile neutrinos.

In conclusion, we have computed the total flux of sterile neutrinos emitted by the Sun, as well as the solar sterile neutrino spectrum; this could be helpful in looking for sterile signatures by the next generation of solar neutrino detectors.

Acknowledgements The author thanks the Fundação para a Ciência e Tecnologia (FCT), Portugal, for the financial support to the Center for Astrophysics and Gravitation (CENTRA), Instituto Superior Técnico, Universidade de Lisboa, through the Grant no. UID/FIS/00099/2013. Moreover, the author also acknowledges the support given by the Institut d'Astrophysique de Paris during his sabbatical visit.

Open Access This article is distributed under the terms of the Creative Commons Attribution 4.0 International License (http://creativecomm ons.org/licenses/by/4.0/), which permits unrestricted use, distribution, and reproduction in any medium, provided you give appropriate credit to the original author(s) and the source, provide a link to the Creative Commons license, and indicate if changes were made.

Funded by SCOAP ${ }^{3}$.

\section{References}

1. K. Abe, Y. Haga, Y. Hayato et al., Phys. Rev. D 91, 052019 (2015)

2. A. Aguilar, L.B. Auerbach, R.L. Burman et al., Phys. Rev. D 64, 112007 (2001)

3. A.A. Aguilar-Arevalo, C.E. Anderson, S.J. Brice et al., Phys. Rev. Lett. 105, 181801 (2010)

4. A.A. Aguilar-Arevalo, B.C. Brown, L. Bugel et al., Phys. Rev. Lett. 110, 161801 (2013)

5. S. Ando, A. Kusenko, Phys. Rev. D 81, 113006 (2010)

6. J.N. Bahcall, B.R. Holstein, Phys. Rev. C 33, 2121 (1986)

7. G. Barenboim, O.M. Requejo, C. Quigg, Phys. Rev. D 71, 083002 (2005)

8. J. Behrens, P.C.-O. Ranitzsch, M. Beck et al., Eur. Phys. J C 77, 410 (2017)

9. J. Bergström, M.C. Gonzalez-Garcia, M. Maltoni, T. Schwetz, J. High Energy Phys. 9, 200 (2015)

10. S. Betts, W.R. Blanchard, R.H. Carnevale, et al., (2013). arXiv: 1307.4738

11. S. Bilenky, in Lecture Notes in Physics (Springer, Berlin, 2010), p. 817

12. J. Billard, L.E. Strigari, E. Figueroa-Feliciano, Phys. Rev. D 91, $095023(2015)$

13. A. Boyarsky, D. Iakubovskyi, O. Ruchayskiy, Phys. Dark Univ. 1, $136(2012)$

14. M. Cirelli, G. Marandella, A. Strumia, F. Vissani, Nucl. Phys. B 708, 215-267 (2005) 
15. A.G. Cocco, G. Mangano, M. Messina, J. Phys. Conf. Ser. 110, 082014 (2008)

16. K. Dolde, S. Mertens, D. Radford et al., Nucl. Instrum. Methods Phys. Res. A 848, 127 (2017)

17. M.C. Gonzalez-Garcia, M. Maltoni, T. Schwetz, Nucl. Phys. B 908, 199 (2016)

18. M.C. Gonzalez-Garcia, M. Maltoni, T. Schwetz, J. High Energy Phys. 11, 52 (2014)

19. M.C. Gonzalez-Garcia, M. Maltoni, Phys. Rep. 460, 1 (2008)

20. C. Giunti, M. Laveder, Phys. Rev. C 83, 065504 (2011)

21. C. Giunti, M. Laveder, Y.F. Li, H.W. Long, Phys. Rev. D 88, 073008 (2013)

22. P.C. de Holanda, A.Y. Smirnov, Phys. Rev. D 69, 113002 (2004)

23. G.-Y. Huang, S. Zhou, Phys. Rev. D 94, 116009 (2016)

24. M.G. Aartsen et al., Phys. Rev. D 95, 112002 (2017)

25. Y.F. Li, Z.-Z. Xing, Phys. Lett. B 695, 205 (2011)

26. Y.F. Li, Z.-Z. Xing, J. Cosmol. Astropart. Phys. 8, 006 (2011)

27. A.J. Long, C. Lunardini, E. Sabancilar, J. Cosmol. Astropart. Phys. 8, $038(2014)$

28. I. Lopes, Phys. Rev. D 95, 015023 (2017)

29. I. Lopes, Phys. Rev. D 88, 045006 (2013)

30. I. Lopes, J. Silk, Mon. Not. R. Astron. Soc. 435, 2109 (2013)

31. I. Lopes, S. Turck-Chièze, Astrophys. J. 765, 14 (2013)

32. R. Adhikari, M. Agostini, N.A. Ky et al., J. Cosmol. Astropart. Phys. 1, 025 (2017)

33. Y.J. Ko, B.R. Kim, J.Y. Kim et al., Phys. Rev. Lett. 118, 121802 (2017)
34. J. Kopp, P.A.N. Machado, M. Maltoni, T. Schwetz, J. High Energy Phys. 5, 50 (2013)

35. T.K. Kuo, J. Pantaleone, Rev. Mod. Phys. 61, 937-980 (1989)

36. M. Maltoni, A.Y. Smirnov, Eur. Phys. J. A 52, 87 (2016)

37. P. Minkowski, Phys. Lett. B 67, 421 (1977)

38. Z. Maki, M. Nakagawa, S. Sakata, Prog. Theor. Phys. 28, 870 (1962)

39. G. Mention, M. Fechner, T. Lasserre et al., Phys. Rev. D 83, 073006 (2011)

40. A. Palazzo, Phys. Rev. D 83, 113013 (2011)

41. A.M. Serenelli, W.C. Haxton, C. Peña-Garay, Astrophys. J. 743, $24(2011)$

42. R.E. Shrock, Phys. Lett. B 96, 159 (1980)

43. S. Turck-Chièze, A. Palacios, J.P. Marques, P.A.P. Nghiem, Astrophys. J. 715, 1539 (2010)

44. S. Weinberg, Phys. Rev. 128, 1457 (1962)

45. M. Yoshimura, N. Sasao, M. Tanaka, Phys. Rev. D 91, 063516 (2015)

46. W.T. Winter, S.J. Freedman, K.E. Rehm, J.P. Schiffer, Phys. Rev. C 73, 025503 (2006)

47. L. Wolfenstein, Phys. Rev. D 17, 2369 (1978) 